\title{
Trends of research articles in the Korean Journal of Medical Education by social network analysis
}

\author{
Hyo Hyun Yoo ${ }^{1}$ and Sein Shin $^{2}$
}

\author{
${ }^{1}$ Department of Medical Education, Chonbuk National University Medical School, and ${ }^{2}$ Division of Science Education, \\ Chonbuk National University College of Education, Jeonju, Korea
}

\section{사회네트워크 분석을 활용한 『한국의학교육』 학회지의 연구 동향 분석}

${ }^{1}$ 전북대학교 의학전문대학원 의학교육실, ${ }^{2}$ 전북대학교 사범대학 과학교육학부

\section{유효현 ${ }^{1}$, 신세인 ${ }^{2}$}

Purpose: This aim of this study is to examine trends in medical education research in the Korean Journal of Medical Education (KJME) and suggest improvements for medical education research.

Methods: The main variables were keywords from research papers that were published in KJME. Abstracts of papers $(n=499)$ that were published from 1991 through 2015 were analyzed by social network analysis (NetMiner 4.0) a common research method for trends in academic subjects.

Results: The most central keywords were "medical education," "clinical competence," "medical student," and "curriculum." After introduction into graduate medical school, newly appearing keywords were "professional behavior," "medical humanities," "communication," and "physician-patient relation." Based on these results, we generated a schematic of the network, in which the five groups before introduction to graduate medical school expanded to nine groups after introduction.

Conclusion: Medical education research has been improving qualitatively and quantitatively, and research subjects have been expanded, subdivided, and specific. While KJME has encompassed medical education studies comprehensively, studies on medical students have risen in number. Thus, the studies that are published in KJME were consistent with the direction of journal and a new study on the changes in medical education is being conducted.

Key Words: Social network analysis, Keywords, Korean Journal of Medical Education, Research trends

\section{서론}

의학교육은 의학교육인증평가 시행, 의학전문대학원(이하
의전원) 체제 도입, 의사국가고시 실기시험 도입 등과 같은 시대적 요구를 반영하며 함께 변화하여 왔다. 특히 의전원은 21세기 지식기반사회가 요구하는 다양하고 폭넓은 학문적 소 양과 자질을 가진 양질의 전문 의료인, 의과학자 및 의학 관련
Received: May 6, 2015 • Revised: June 5, 2015 • Accepted: June 8, 2015 Corresponding Author: Sein Shin (http://orcid.org/0000-0002-8333-7375)

Division of Science Education, Chonbuk National University College of Education, 567 Baekje-daero, Deokjin-gu, Jeonju 54896, Korea

Tel: +82.63.270.2778 Fax: +82.63.270.2783 email: sein3027@gmail.com
Korean J Med Educ 2015 Dec; 27(4): 247-254.

http://dx.doi.org/10.3946/kjme.2015.27.4.247

eISSN: 2005-7288

(C) The Korean Society of Medical Education. All rights reserved. This is an open-access article distributed under the terms of the Creative Commons Attribution Non-Commercial License (http:// creativecommons.org/licenses/by-nc/3.0/), which permits unrestricted non-commercial use, distribution, and reproduction in any medium, provided the original work is properly cited. 
Hyo Hyun Yoo and Sein Shin: Research trends in medical education through social network analysis

분야의 지도자를 배출하기 위한 목적으로 도입되었다[1]. 의 전원 도입이라는 교육체제 변화는 교육 목표, 교육 과정, 교육 방법, 교육 평가 등 교육의 전 과정에 새로운 변화를 가져왔 다. 특히 교육 대상인 학생이 고등학교를 졸업하고 입학한 의 과대학생에서 대학교를 졸업하고 입학한 의학전문대학원생 으로 바뀌었다. 이러한 의학교육 체제의 변화, 사회적 요구와 정책적 변화 등은 학술지 연구 동향의 변화와 밀접한 관련이 있을 것이다.

일반적으로 특정 학문 혹은 분야의 연구 동향을 분석하는 연구는 기존의 연구 성과를 점검하여 현재 상태를 파악하고 미래의 연구 방향을 제시하는 데 목적이 있다[2]. 즉, 연구 동 향 연구는 해당 분야의 연구 주제와 방법 등이 과거로부터 지 금까지 어떤 경향을 보이면서 이루어지고 있는지에 대한 이 해를 가능하게 하여 그 분야의 지식기반 구축을 공고히 하는 데 용이하고, 해당 분야의 현실적인 요구에 대응하도록 연구 의 방향을 조정하거나 새롭게 설정하는 데 도움을 준다[3]. 연구 동향을 분석한 연구들은 특정 학문 분야의 학술지 전반 을 대상으로 연구하거나, 특정 학문 분야 내의 특정 영역을 대 상으로 분석하는데, 본 연구는 『한국의학교육(Korean Journal of Medical Education)』이라는 학술지만의 연구 동향을 분석 하였다. 그 이유는 단일 학술지 연구 동향 연구는 게재된 연구 물들이 얼마나 학술지가 지향하는 편집방향에 부합하는지를 판단하는 데 도움을 주고, 편집방향에 부합되는 분야의 현실 적인 요구와 흐름을 적절하게 반영하고 있는지를 분석적으로 판단하는 데 도움을 주기 때문이다[4].

의학교육분야의 대표적인 학술지 『한국의학교육』은 1989 년 창간호를 시작으로 2015년 현재 27권 1호까지 발간되었 다. 2004년도에 한국학술진흥재단 등재후보 학회지가 되었 고, 2007년 등재지로 인정받았으며, 더 나아가 국제기준에 부 합하는 국제 학술지로서 인정받는 것을 목표로 준비하고 있 다[5]. 또 한 번의 큰 도약을 준비하기 위해 그동안 축적되어 온 연구 동향을 분석하고 추후 연구들을 조망해보는 것은 의 미 있는 노력이라고 생각한다.

기존의 연구 동향과 관련된 대부분 연구들의 연구 방법은 사전에 일정한 분류 준거를 설정해두고, 분류 준거에 연구 성 과들을 분류하는 방식으로 분석하였다. 의학교육과 관련된 국내외 연구들도 학문의 특성을 반영하여 설정한 개념적 분
석틀이나 일정 분류체계를 준거로 해당 범주에 해당하는 논 문의 수를 빈도 등으로 분석하는 정량적 접근방법을 주로 사 용하였다[6,7,8,9]. 이 연구방법은 주요 개념이나 미리 정해진 분류주제의 출현 빈도를 통해 특정 주제가 얼마나 다루어지 고 있는지 파악할 수 있지만, 그것들이 어떤 맥락에서 다루어 지고 있고 이들 간의 관계는 어떠한지를 파악하는 것에는 한 계가 있다. 따라서 국내외 많은 학문 분야의 연구 동향 관련 연구들에서는 총체적 관점에서 다양한 접근 방법 등을 사용 하여 다각적으로 분석하고 그 결과를 토대로 학문적 정체성 확보 및 발전과제를 도출하기 위한 시도가 지속적으로 이루 어져 왔다[10]. 사회과학연구에서는 연구 동향을 분석하기 위 하여 사회네트워크 분석법을 활용하는 연구가 이어지고 있다 [11,12]. 사회네트워크 분석은 분석 대상들 간의 관계에 기반 을 둔 분석으로 분석 대상의 구조적 정량지표를 제시하고, 요 소들 간에 관계를 맥락적으로 이해할 수 있는 접근방식이다 [13]. 사회네트워크 분석은 사람, 사물 등의 관계 외에도 논문 이나 신문 기사와 같은 텍스트(text)를 대상으로도 활발히 이 루어지고 있다. 이는 하나의 텍스트가 여러 단어들 사이의 관 계를 통해 형성된 네트워크라는 것을 전제로 한다[14]. 연구 동향도 여러 연구 주제들로 이루어진 네트워크로 볼 수 있기 때문에 최근 논문의 주제어 간 구조적 관계성에 초점을 두고 연구 동향을 파악하는 연구에서 사회네트워크 분석을 많이 활용하고 있다[15].

따라서 본 연구의 목적은 『한국의학교육』에 게재된 논문들 을 바탕으로 사회네트워크 분석을 사용하여 의전원 도입 전 후의 연구 변화를 도출하고, 이를 바탕으로 의학교육의 발전 을 위한 방향을 제시하고자 한다.

\section{대상 및 방법}

\section{1. 대상}

본 연구는 국내 의학교육 분야의 대표적 학술지인 『한국의 학교육』에 게재된 논문의 초록에 제시되어 있는 주제어들 (keywords)을 대상으로 하였다. 한국의학논문데이터베이스 (Korean Medical Database)에서 제공되는 『한국의학교육』 
에 게재된 논문(종설, 단신, 원저 등)의 서지데이터에서 주제 어만을 연구 대상으로 하였다.

수집 대상 논문의 시간적 범위는 한국의학논문데이터베이 스에『한국의학교육』의 주제어가 등록되기 시작한 시점인 1991년부터 2015년(27권 1호)까지로 한정하였다. 의전원 체 제는 2005년에 도입되었으나, 전반적으로 확대 도입시점 2006년을 기준으로 대상 시기를 1999 2005년, 2006 2015년 으로 구분하였다.

1991 2005년 시기에 발간된 학술지에 게재된 225편 논문 에서는 중복 단어를 포함하여 690개의 주제어가 나타났고, 2006 2015년(27권 1호)까지의 학술지에 게재된 274편 논문 에서는 중복 단어를 포함하여 1,006 개의 주제어가 나타났다. 총 499편 논문에서 제시한 1,696 개의 주제어를 분석 대상으 로 하였다.

\section{2. 방법}

수집한 자료의 분석은 자료의 전처리 단계, 네트워크 생성, 분석 및 도식화 단계로 구분하여 실시하였고, 이를 위하여 네트 워크분석 전문프로그램인 NetMiner 4.0 (Cyram Inc., Seoul, Korea)을 사용하였다.

\section{1) 전처리 단계}

수집된 전체 주제어 중 students와 student, problembased learning과 problem based learning, 그리고 CPX와 clinical performance examination과 같이 단수. 복수, 하이 픈 연결, 약어 등으로 형태는 다르지만 같은 의미를 나타내는 주제어들은 하나의 동일한 형태의 단어로 정리하였다. 이후 중심적인 연구 동향을 확인하기 위하여 논문에 두 번 이상 사 용된 주제어만을 선별하였다. 그 결과 의전원 도입 전 82개, 도입 후 125 개, 총 207 개의 고유 주제어를 추출하였다.

\section{2) 네트워크 생성 단계}

전처리 단계에서 정리된 자료는 '논문×주제어' 행렬의 이 원모드 네트워크(2-mode network)이므로 주제어 간의 관계 만을 분석하기 위해 주제어들의 공출현(Co-occurrence) 빈 도에 기초하여 ‘주제어×주제어' 행렬의 일원모드 네트워크 (1-mode network)로 변환하였다. 공출현 빈도는 특정 주제 어가 유사한 맥락을 가진 주제어들이 함께 사용되는 빈도로 네트워크상에서 연결의 가중치(weight)로 산출된다[13]. 즉,
여러 논문에 동시 출현하는 주제어 간에는 높은 연결 가중치 가 나타난다.

\section{3) 네트워크 분석 단계}

네트워크 분석 방법 중 하나의 특정 개념이 전체 네트워크 에서 중앙에 위치하는 정도를 표현하는 중심성(centrality) 분석 방법을 사용하였는데, 그 이유는 전체적인 네트워크 구 조에서 주제어가 어떠한 위치에 있고 다른 주제어들과 관계 에서 중요한 위치를 차지하는 정도를 알 수 있기 때문이다. 중 심성은 연결정도중심성, 인접중심성, 사이중심성, 위세중심성 의 네 가지 유형으로 구분된다. 본 연구에서는 구조적 정량지 표를 바탕으로 의전원 도입 전후의 네트워크가 어떠한 구조 적 차이를 보이는지 분석하기 위하여 특정 주제어가 직접적 으로 연결되어 있는 다른 주제어들과 어느 정도의 관계를 맺 고 있는가를 의미하는 연결정도중심성[13]을 분석하였다. 네 트워크 내에서 중심적이고 활발하게 연구되는 주제어일수록 연결정도중심성은 높다[16].

\section{4) 네트워크 도식화 단계}

네트워크상의 중심적 주제어들을 대상으로 구조적 관계를 거시적으로 분석하기 위해 네트워크는 연결 가중치가 0.2 이 상인 연결을 지니는 주제어들을 선별하였다. 네트워크상에서 개별 주제어들은 점(nodes), 하나의 논문에 사용된 주제어들 간의 관계는 선(links)으로 설정하여 도식화하였다. 도식화된 네트워크를 탐색적으로 분석하여 의전원 도입 전후로 나타난 변화를 보다 맥락적으로 이해하고자 주제어들을 군집화하였 다. 도식화된 네트워크에서 점의 크기는 연결정도중심성에 비례하도록 하였고, 군집을 나타내는 경계선은 점선으로 표 시하였다.

\section{결과}

\section{1. 빈도 분석}

네트워크 분석에 앞서 논문에 나타난 빈도 상위 20위 내의 주제어를 확인하였다(Table 1). 의전원 도입 전의 논문에서는 medical education이 30회(15.3\%)로 가장 많이 나타났으며, problem-based learning 28회(14.3\%), evaluation 23회 
Table 1. Frequency of Keywords in KJME before and after Graduate Medical School

\begin{tabular}{|c|c|c|c|c|}
\hline \multirow{2}{*}{ No. } & \multicolumn{2}{|l|}{ Before (1999-2005) } & \multicolumn{2}{|l|}{ After (2006-2015) } \\
\hline & Keywords & Frequency $(\%)$ & Keywords & Frequency $(\%)$ \\
\hline 1 & Medical education & $30(15.3)$ & Medical student & $70(22.7)$ \\
\hline 2 & Problem-based learning & $28(14.3)$ & Medical education & $32(10.4)$ \\
\hline 3 & Evaluation & $23(11.7)$ & Clinical competence & $31(10.0)$ \\
\hline 4 & OSCE & $13(6.6)$ & Educational measurement & $23(7.4)$ \\
\hline 5 & Clinical clerkship & $12(6.1)$ & Communication & $16(5.2)$ \\
\hline 6 & Medical student & $10(5.1)$ & Problem-based learning & $14(4.5)$ \\
\hline 7 & Curriculum & $8(4.1)$ & Curriculum & $13(4.2)$ \\
\hline 8 & Item analysis & $7(3.6)$ & Physician-patient relation & $12(3.9)$ \\
\hline 9 & Medical ethics & $7(3.6)$ & Clinical clerkship & $11(3.6)$ \\
\hline 10 & Medical school & $7(3.6)$ & Clinical performance examination (CPX) & $11(3.6)$ \\
\hline 11 & Standardized patient & $7(3.6)$ & Education & $10(3.2)$ \\
\hline 12 & Education & $6(3.1)$ & Teaching & $10(3.2)$ \\
\hline 13 & Validity & $6(3.1)$ & Program evaluation & $9(2.9)$ \\
\hline 14 & Clinical competence & $5(2.6)$ & Medical school & $8(2.6)$ \\
\hline 15 & Clinical performance examination (CPX) & $5(2.6)$ & Personality & $7(2.3)$ \\
\hline 16 & Curriculum development & $5(2.6)$ & Premedical student & $7(2.3)$ \\
\hline 17 & Internet & $5(2.6)$ & Undergraduate medical education & $7(2.3)$ \\
\hline 18 & Clinical skill & $4(2.0)$ & Academic achievement & $6(1.9)$ \\
\hline 19 & Correlation & $4(2.0)$ & Communication skill & $6(1.9)$ \\
\hline \multirow[t]{2}{*}{20} & Educational objectives & $4(2.0)$ & Medical professionalism & $6(1.9)$ \\
\hline & Total & $196(100.0)$ & Total & $309(100.0)$ \\
\hline
\end{tabular}

KJME: Korean Journal of Medical Education.

(11.7\%), OSCE 13회(6.6\%), clinical clerkship 12회(6.1\%), medical student 10 회(5.1\%) 순으로 나타났다. 의전원 도입 후의 논문에서는 medical student가 70회(22.7\%)로 가장 많 이 나타났으며, medical education 32회(10.4\%), clinical competence 31회(10.0\%), educational measurement 23회 (7.4\%), communication 16회(5.2\%) 순으로 나타났다. 의전 원 도입 전에 비하여 도입 후에 논문수가 증가함 점을 고려하 더라도, medical student, clinical competence와 관련된 주 제어가 크게 증가하는 추세를 보였다.

\section{2. 연결정도중심성 분석}

의전원 도입 전과 후의 논문들에 사용된 주제어들 중 연결정 도중심성이 가장 높은 20 개의 주제어를 분석한 결과(Table 2), 의전원 도입 전에는 medical education이 0.0397로 연결중심 성이 가장 높게 나타났고, evaluation, standardized patient, clinical competence, OSCE, clinical performance examination (CPX)순으로 나타났다 $(0.0394,0.0318,0.0316,0.0310$,
0.0291). 의전원 도입 후에는 medical student가 0.0551로 연 결정도중심성이 가장 높게 나타났고, medical education, clinical competence 순으로 나타났다(0.0302, 0.0295).

의전원 도입 전후 medical education, clinical competence, medical student, curriculum이 공통적으로 높은 연 결정도중심성을 나타냈는데, 그 중 medical student는 의전 원 도입 후 연결정도중심성이 약 2 배 이상 높아졌다. 이와 더 불어 상위 20위 내의 높은 연결정도중심성을 보이는 주제어 는 depression, personality type, learning 등 학생의 심리 적, 인지적 특성들과 관련된 주제어로 나타났다.

의전원 도입 후 professional behavior, medical humanities, communication, physician-patient relation 등 전문 직업성이나 의사소통능력과 관련된 주제어들이 새롭게 중심 적인 연구 주제어로 나타났다.

\section{3. 네트워크 도식화를 통한 주제군 분석}

연구 동향을 구조적으로 파악하기 위해 네트워크를 도식화 
Table 2. Degree Centrality of Keywords in KJME before and after Graduate Medical School

\begin{tabular}{rlcllc}
\hline \multirow{2}{*}{ No. } & \multicolumn{2}{c}{ Before (1999-2005) } & & \multicolumn{2}{c}{ After (2006-2015) } \\
\cline { 2 - 3 } \cline { 5 - 6 } 1 & Medical education & Degree centrality & & Keywords & Degree centrality \\
2 & Evaluation & 0.0397 & & Medical student & 0.0551 \\
3 & Standardized patient & 0.0394 & & Medical education & 0.0302 \\
4 & Clinical competence & 0.0318 & & Clinical competence & 0.0295 \\
5 & OSCE & 0.0316 & & Educational measurement & 0.0252 \\
6 & Clinical performance examination & 0.0310 & & Program evaluation & 0.0246 \\
7 & Reliability & 0.0291 & & Teaching & 0.0244 \\
8 & Problem-based learning & 0.0260 & & Professional behavior & 0.0244 \\
9 & Medical student & 0.0241 & & Multi-disciplinary curriculum & 0.0244 \\
10 & Validity & 0.0221 & & Inter-disciplinary curriculum & 0.0244 \\
11 & Curriculum & 0.0218 & & Curriculum & 0.0230 \\
12 & Clinical skill & 0.0216 & & Communication & 0.0228 \\
13 & Correlation & 0.0210 & & Depression & 0.0225 \\
14 & Clerkship & 0.0200 & & Peer review & 0.0224 \\
15 & Item analysis & 0.0199 & & Attitude & 0.0213 \\
16 & Medical school & 0.0197 & & Physician-patient relation & 0.0206 \\
17 & Faculty track system & 0.0195 & & Personality type & 0.0204 \\
18 & Internet & 0.0192 & & Medical humanities & 0.0201 \\
19 & Instruction evaluation & 0.0186 & Academic performance & 0.0198 \\
20 & Pathology & 0.0185 & & Perception & 0.0193 \\
& & 0.0178 & Learning & 0.0192 \\
\hline
\end{tabular}

KJME: Korean Journal of Medical Education.

Fig. 1. Keyword Network and Groups before Introduction into Graduate Medical School

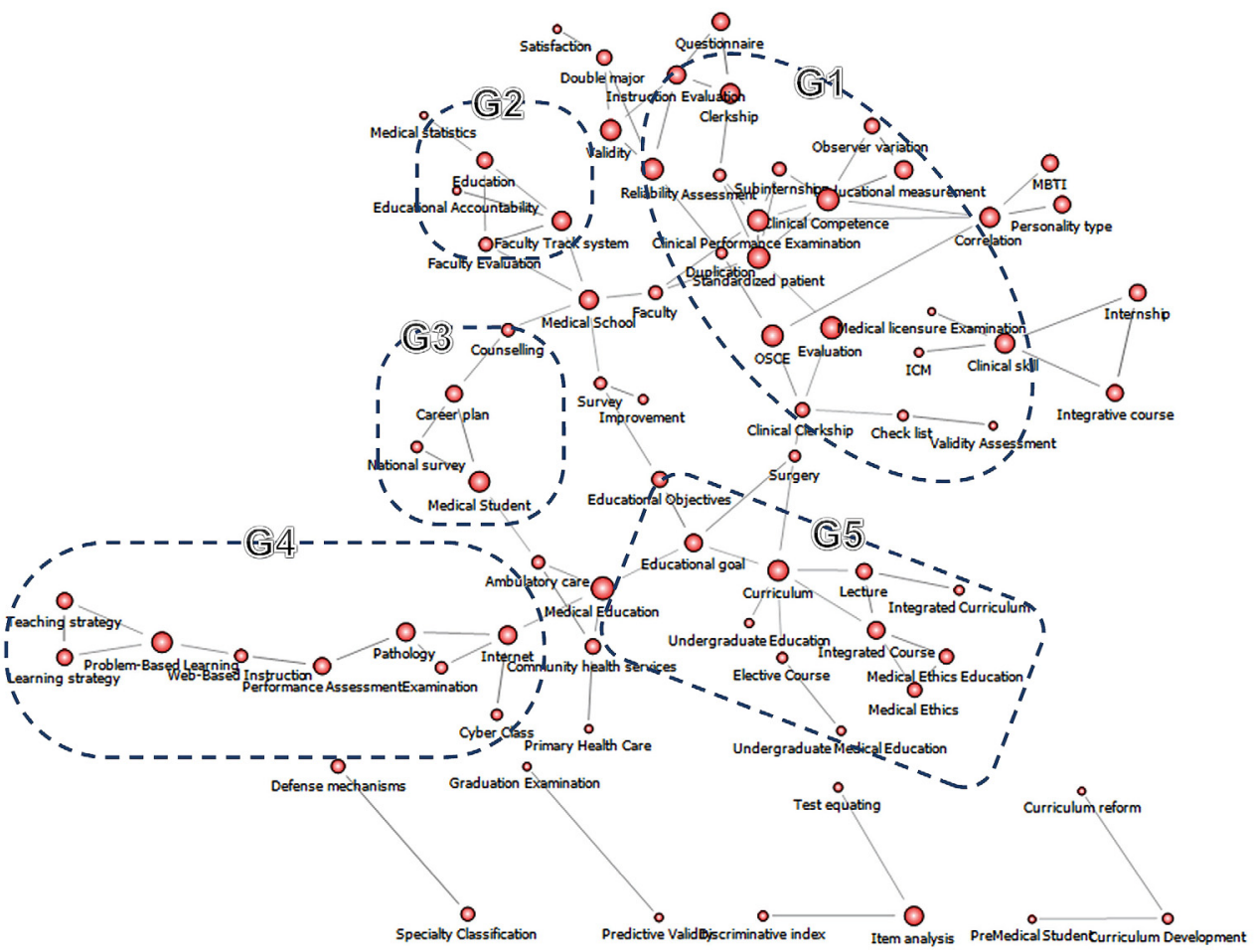


Fig. 2. Keyword Network and Groups after Introduction to Graduate Medical School

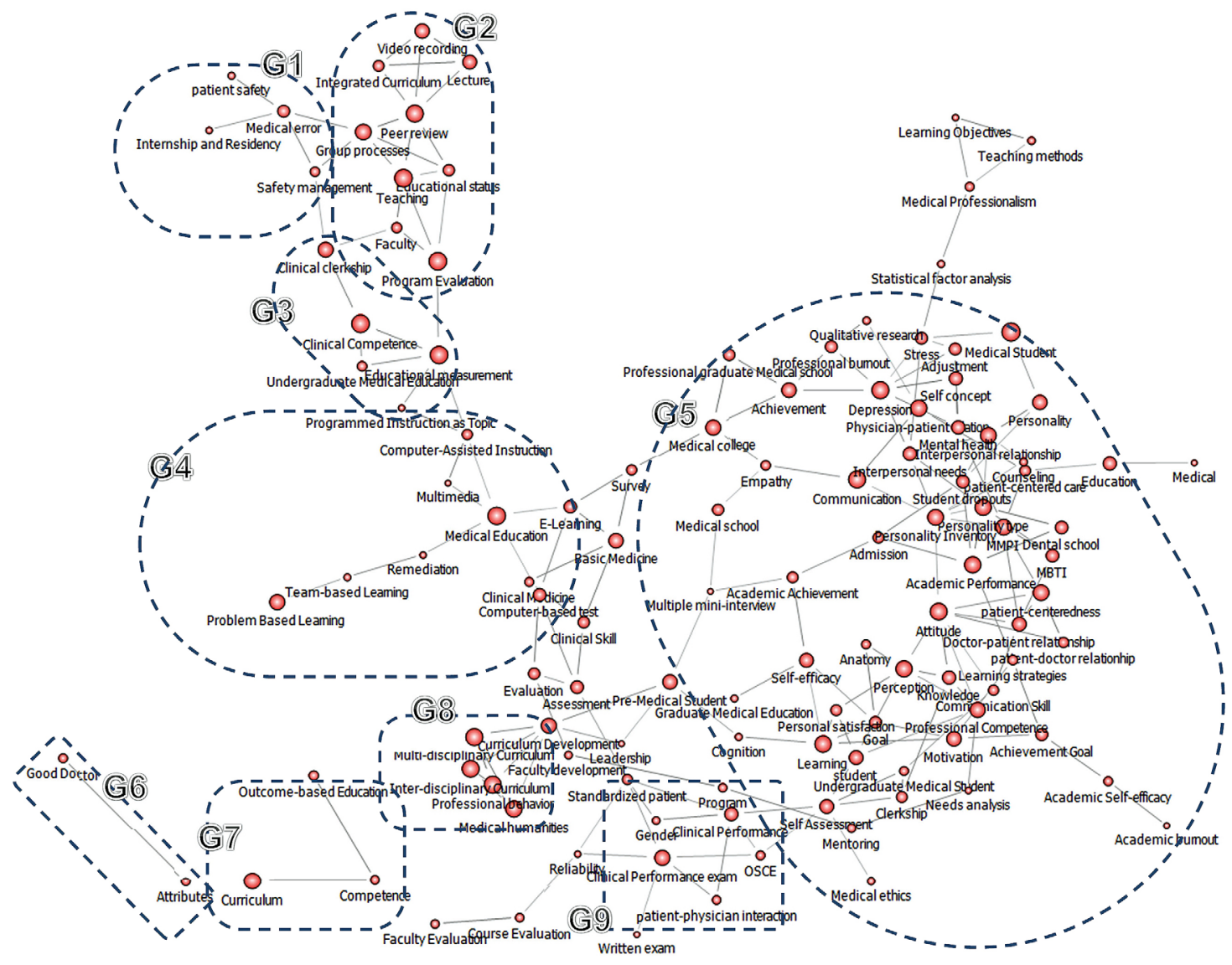

한 결과 의전원 도입 전에는 5개의 군집을 도출할 수 있었고 (Fig. 1), 도입 후에는 9개의 군집을 도출할 수 있었다(Fig. 2). 의전원 도입 전에는 $\mathrm{CPX}, \mathrm{OSCE}$ 와 관련된 주제군(clinical competence, standardized patient 등) (Fig. 1; G1), 교수평 가와 관련된 주제군(faculty evaluation 등) (Fig. 1; G2), 학 생과 관련된 주제군(career plan, counselling 등) (Fig. 1; $\mathrm{G} 3)$, 교육방법과 관련된 주제군(problem-based learning, web-based instruction 등) (Fig. 1; G4), 교육과정과 관련된 주제군(integrated curriculum, elective course 등) (Fig. 1; G5)이 나타났다.

의전원 도입 후에는 환자 안전과 관련된 주제군(safety management, medical error 등) (Fig. 2; G1), 강의 평가와 관련된 주제군(video record, program evaluation 등) (Fig. 2; G2), 임상실습과 관련된 주제군(clinical clerkship, clinical competence) (Fig. 2; G3), 교육 방법과 관련된 주 제군(team-based learning, e-learning 등) (Fig. 2; G4), 학
생과 관련된 주제군(self-efficacy, motivation 등) (Fig. 2; $\mathrm{G5})$, 인재상과 관련된 주제군(good doctor 등) (Fig. 2; G6), 교육 패러다임과 관련된 주제군(outcome-based education 등) (Fig. 2; G7), 교육 과정과 관련된 주제(curriculum development 등) (Fig. 2; G8), $\mathrm{CPX}, \mathrm{OSCE}$ 와 관련된 주제 군(clinical performance, standardized patient 등) (Fig. 2; G9)이 나타났다.

의전원 도입 후 환자 안전, 강의 평가, 인재상, 교육 패러다 임과 관련된 주제군이 네트워크상에 새롭게 추가되었고, 학 생과 관련된 주제군의 점과 선이 더욱 증가하여 네트워크상 에서 큰 면적을 차지하고 있는 것으로 나타났다.

\section{고찰}

본 연구의 목적은 『한국의학교육』에 게재된 논문들을 사회 
네트워크 분석을 통해 의전원 도입 전후의 비교를 통하여 연구 동향을 체계적으로 분석함으로써 현재까지의 학문적 발전 수 준과 특성을 파악하고, 향후 의학교육의 연구 방향을 탐색하기 위한 것이다. 연구 결과를 요약하고 논의하면 다음과 같다.

첫째, 의전원 도입 전에는 종래부터 운영되었던 교육 과정, 교육 방법, 평가 등 의학교육 전반의 시스템 관리와 개선 등과 관련된 연구가 주로 이루어진 반면, 의전원 도입 후에는 교육 대상인 학생과 관련된 요인들의 연구가 현저히 증가하는 추 세가 나타났다. 즉, 의전원 도입 전에는 의학교육에 대한 기초 적 접근 연구가 다양한 측면에서 이루어진 반면 도입 후에는 학생에 대한 인지적, 정의적 특성과 같은 교육심리적 접근의 연구가 활발히 이루어지고 있다. 의전원 도입 후 학생 배경의 변화로 학생에 대한 관심이 증가한 점과 이런 학생들의 특성 들을 다양한 시각으로 분석할 수 있는 의학교육 연구자들이 연구에 활발하게 참여하게 된 점과 관련이 있을 것으로 사료 된다. 의학교육이 지향하는 학습자 중심의 교육은 학습자에 대한 이해로부터 시작된다. 학습자 특성을 고려한 심도 있는 연구의 증가는 기존의 교수자 중심 교육에서 학습자 중심 교 육으로 이행하고 있음을 단편적으로 보여준다. 따라서 향후 의학교육 관계자들이 학생들을 이해하고 보다 효과적으로 가 르치기 위해서는 학생 특성에 대한 연구가 지속적으로 이루 어져야 할 것이다.

둘째, 의전원 도입 전보다 후에 연구 주제군이 보다 다양해 지고, 주제어들이 세분화되는 것으로 나타났다. 이 결과는 1989년부터 2010년까지 『한국의학교육』 학술지를 통시적 분 석에 따라 분석한 결과 연구 주제면에서 소주제 영역의 다양 화와 세분화 추세가 있다는 선행 연구와 분류기준은 다르지 만 추세 변화는 일치하였다[9]. 네트워크 분석 결과, 의전원 도입 전에 $\mathrm{CPX}$ 와 OSCE를 포함한 임상진료역량, 교수 평가, 학생, 교수 방법, 교육 과정과 관련된 주제군을 중심으로 연구 가 이루어진 반면, 의전원 도입 후에는 환자 안전, 강의 평가, 인재상, 교육 패러다임과 같은 새로운 주제군이 더 추가되면 서 연구 주제들이 다양화되었음을 확인할 수 있었다. $\mathrm{CPX}$ 와 OSCE를 포함한 임상진료역량이나 평가와 관련된 주제어의 연결정도중심성은 의전원 도입 전과 후 모두 높게 나타나 의 학교육에서 중요한 연구 주제 중 하나임을 알 수 있다. 최근 의사국가고시의 실기시험 도입과 더불어 지식 위주보다는 실
행능력을 겸비한 의사를 양성하고자 하는 의학교육의 변화 등에 따라 향후 임상진료역량과 관련된 전문적인 연구가 필 요할 것이다. 교수 평가와 관련된 주제군은 의전원 도입 전에 는 교육, 교육 분야 책무성 등의 주제어를 사용하였지만 의전 원 도입 후에는 교수자와 관련된 주제어들의 사용이 저조한 것으로 나타났다. 교육의 전반적인 운영은 교수의 역량에 의 해 영향을 받기 때문에 변화하는 교육 방법과 교육 평가 등에 대한 교수들의 역량 강화를 목적으로 하는 교육 프로그램 개 발과 그 효과를 분석하는 등의 연구들이 보다 활발히 이루어 질 필요가 있다고 판단된다.

셋째, 의학교육의 현실적인 요구와 흐름을 반영한 연구들 이 이루어지고 있는 것으로 나타났다. 사회적 요구를 반영한 성과바탕교육이라는 세계 의학교육 패러다임의 변화, 좋은 의사라는 인재상, 의료인문학과 의사소통을 포함한 전문직업 성 등 새로운 주제의 연구가 진행되고 있으나 아직 기초적인 수준에서 진행되고 있음을 네트워크를 통해 확인하였다. 따 라서 향후 이와 같은 주제와 관련하여 보다 심층적인 연구들 이 이루어질 필요가 있다. 연구와 교육현장의 상호 소통에 의 한 연구 주제의 반영은 교육과 연구가 선순환되는 구조로써 의학교육 발전을 위해서는 반드시 필요하다. 향후의 의학교 육 연구에 있어서도 의학교육 관련 연구자들은 의학교육 현 장의 다양한 문제들을 시기적절하게 반영함과 동시에 대안을 제시함으로써 의학교육의 발전을 도모하는 견인차 역할을 담 당해야 할 것이다.

현재까지『한국의학교육』 학술지에 게재된 논문들은 학술 지가 지향하는 학문 분야의 정체성에 부합되고, 의학교육의 정책과 현안, 새로운 추세나 현상을 고려한 연구들이 진행되 고 있음을 확인할 수 있었다. 이와 더불어 향후에도 의학교육 발전에 기여할 수 있는 이론과 실제적 지식을 기반으로 의학 교육의 비전을 제시하기 위해 지속적인 노력과 관리 등을 하 는 것이 『한국의학교육』 학술지가 지향해야 할 방향이라고 사료된다.

본 연구에서 구성된 네트워크는 연구자 자신의 인식에 의 해 선택된 주제어들로 구성된 것이므로 의학교육의 실제 학 문구조와는 다소 상이할 수 있다는 한계점을 지닌다. 추후에 는 다양한 분석 방법을 통해 다각적으로 학술지의 연구 동향 을 조사하고, 시기를 보다 세분화하여 네트워크를 비교하거나 
Hyo Hyun Yoo and Sein Shin: Research trends in medical education through social network analysis

국내외 의학교육 관련 학술지의 연구 동향을 비교하는 등의 연구를 한다면 연구 동향의 동역학적 변화를 더 명확히 볼 수 있을 것이다. 아울러 본 연구의 결과를 기존의 선행 연구와 통 합하고 향후 축적되는 자료들을 활용하여 학회지의 국제화 전 략을 추진하는 데 필요한 기초자료로 활용되기를 기대해본다.

Acknowledgements: None.

Funding: None.

Conflicts of interest: None.

\section{REFERENCES}

1. Huh KB. The necessity of professional graduate medical school. J Korean Med Assoc 2001; 44: 1034-1038.

2. Byun KY, Ka SH. Critical reflections on higher education administration research in Korea: an analysis of research topic, method, and researcher. J Educ Adm 2012; 30: 135-160.

3. Donmoyer R, Imber M, Scheurich JJ. The knowledge base in educational administration: multiple perspectives. New York, USA: State University of New York Press; 1995.

4. Shin HS, Ka SH, Lee K, Joo YH. Analysis of research trends in The Journal of Korean Education: 1974-2012. J Korean Educ 2013; 40: 207-238.

5. Kim S. Twenty years of the Korean Journal of Medical Education: past, present, and future. Korean J Med Educ 2009; 21: 1-2.

6. Regehr G. Trends in medical education research. Acad
Med 2004; 79: 939-947.

7. Obeidat AS, Alhaqwi AI, Abdulghani HM. Reprioritizing current research trends in medical education: a reflection on research activities in Saudi Arabia. Med Teach 2015; 37 Suppl 1: S5-S8.

8. Kim S. Current trends in medical education by analysis of journals of medical education. Korean J Med Educ 2004; 16: 109-117.

9. Lee YH, Lee YM, Kwon H. Trends analysis on research articles in the Korean Journal of Medical Education. Korean J Med Educ 2012; 24: 287-299.

10. Lee MH, Kim EJ. Research trends in educational journal articles: content analysis. J Korean Biblia Soc Libr Inf Sci 2011; 22: 81-100.

11. Cho J. A study for research area of library and information science by network text analysis. J Korean Soc Inf Manage 201 1; 28: 65-83.

12. Yang SD. Semantic network analysis of research trend related to private security. J Korean Contents Assoc 2013; 13: 894-901.

13. Son DW. Social network analysis. Seoul, Korea: Kyungmoonsa; 2002.

14. Sowa JF. Conceptual structures: information processing in mind and machine. Reading, USA: Addison-Wesley; 1984.

15. Lee SK, Jeong S, Kim HG, Yom YH. A social network analysis of research topics in Korean nursing science. J Korean Acad Nurs 2011; 41: 623-632.

16. Wasserman S, Faust K. Social network analysis: methods and applications. Cambridge, UK: Cambridge University Press; 1994 